\title{
DETERMINATION OF VARIANCE OF TIME TO RECRUITMENT WITH INTER-DECISION TIME AS GEOMETRIC PROCESS WHEN THE THRESHOLD HAS THREE COMPONENTS
}

\author{
${ }^{1}$ A. DEVI \\ ${ }^{1}$ Assistant Professor, PG \& Research Department of Mathematics, Bishop Heber College, \\ Tiruchirappalli-620 017, Tamil Nadu, India. \\ ${ }^{2}$ B. SATHISH KUMAR \\ ${ }^{2}$ Assistant Professor, PG \& Research Department of Mathematics, Bishop Heber College, \\ Tiruchirappalli-620 017, Tamil Nadu, India
}

\begin{abstract}
In this paper, the problem of time to recruitment is analyzed for a single grade manpower system using an univariate CUM policy of recruitment. Assuming policy decisions and exits occur at different epochs, wastage of manpower due to exits form a sequence of independent and identically distributed exponential random variables, the inter-decision times form a geometric process and inter-exist time form an independent and identically distributed random variable. The breakdown threshold for the cumulative wastage of manpower in the system has three components which are independent exponential random variables. Employing a different probabilistic analysis, analytical results in closed form for system characteristics are derived.
\end{abstract}

\section{Keywords}

Single grade manpower system, non-instantaneous exits, intensity of attrition, independent wastage of manpower, geometric process, breakdown threshold with three components, univariate CUM policy of recruitment and variance of time to recruitment.

\section{Introduction}

Wastage of personnel due to retirement, death and resignation is a common phenomenon in administrative as well as production oriented organizations. There are certain special problems associated with the organization engaged in sales and marketing. Frequent exits and recruitments are very common in such organizations. Whenever the organization announces revised polices regarding sales target, revision of wages, incentives and perquisites the exodus is possible. Reduction in the total strength of marketing personnel adversely affects the sales turnover of the organization. Frequent recruitments may also be expensive due to the cost of recruitments and training. As the wastage of manpower is unpredictable, a suitable recruitment policy has to be designed to overcome this wastage. The univariate recruitment policy, usually known as CUM policy of recruitment in the literature, is based on the replacement policy associated with the shock model approach in reliability theory and is stated as follows: Recruitment is made whenever the cumulative wastage of man hours exceeds its breakdown 
threshold. Several researchers have studied the problem of time to recruitment for a single grade manpower system using shock model approach. In [1] and [2] the authors have discussed some manpower planning models for a single and multi-grade manpower system using Markovian and renewal theoretic approach. In [13] the authors have analyzed the problem of time to recruitment for the single grade manpower system which is subject to attrition with instantaneous exits, using CUM policy of recruitment when the wastage of manpower process and inter-decision time process are independent. In [11], the author has studied the problem by associating geometric process and order statistics for inter-decision times. In [9], the authors have analyzed the work in [12] with (i) exponential breakdown threshold and (ii) extended exponential threshold having shape parameter 2 using a bivariate CUM policy of recruitment when the inter-decision times form a geometric process. In [10], the authors have studied the problem of time recruitment by assuming that the attrition is generated by a geometric process of inter-decision times. Variance of the time to recruitment for a single grade manpower system with optional and mandatory thresholds is obtained in [16] when inter-decision times form an order statistics. In [17], the authors have obtained variance of time to recruitment when the breakdown threshold level for the cumulative wastage of manpower is the sum of the exponential breakdown threshold levels of wastage and backup resource for manpower using Laplace transform technique. Recently, in [15], the authors have studied the work in [17] when the inter-decision times form a geometric process using a different method employed in [10]. In [3] the authors have considered the single grade manpower system with non-instantaneous exits and obtained variance of the time to recruitment when the wastage of manpower, interdecision times and exit times are independent and identically distributed continuous random variables according as the mandatory breakdown threshold is an exponential random variable or extended exponential random variable with shape parameter 2 or a continuous random variable with SCBZ property. In [6] and [7] the authors have extended the research work in [3] when the inter-decision times form (i) a geometric process and (ii) an order statistics respectively. In [4], [11] and [5] the authors have studied the work in [3], [6] and [7] using the method of [10]. In [8] the authors have discussed when the threshold has two components, The present paper extends the research work in [8] when the breakdown threshold level for the cumulative wastage in the manpower system is the sum of three components namely an exponential threshold for cumulative wastages due to exits, an exponential threshold for cumulative wastage due to frequent breaks of existing workers and an exponential threshold for cumulative wastage due to backup sources.

\section{MODEL DESCRIPTION AND ANALYSIS}

Consider an organization taking policy decisions at random epochs in $(0, \infty)$ and at every decision making epoch a random number of persons quit the organization. There is an associated wastage of manpower, if a person quits. It is assumed that the loss of manpower is linear and cumulative. For $\mathrm{i}=1,2,3 \ldots$, let $\mathrm{X}_{\mathrm{i}}$ be independent and identically distributed exponential random variables representing the amount of depletion of manpower (wastage of man hours) at the $\mathrm{i}^{\text {th }}$ exit epoch with probability distribution function $\mathrm{M}($.$) , and mean \frac{1}{\alpha}(\alpha>0)$. Let $S_{i}$ be the cumulative wastage of manpower in the first $i$ exit epochs and $m_{i}$ be its probability density function. The inter-policy decision times form a geometric process with rate $\mathrm{c}, \mathrm{c}>0$. It is assumed that distribution of $A_{1}$ is hyper-exponential with parameters $a_{1}, \lambda_{1}$ and $\lambda_{2}$. Let $B_{i}$ 
be the time between (i-1) $)^{\text {th }}$ and $i^{\text {th }}$ exit epochs, forming a sequence of independent and identically distributed random variables with probability distribution function $\mathrm{G}($.) and density function $\mathrm{g}($.$) . Let D_{i+1}$ be the waiting time upto $(\mathrm{i}+1)^{\text {th }}$ exit epoch.Let $\mathrm{Y}$ be the breakdown threshold for the cumulative wastage of manpower in the organization with probability density function $\mathrm{h}($.$) . It is assumed that sum of the exponential breakdown threshold level \mathrm{Y}_{1}$ for cumulative wastage due to exits with mean $\frac{1}{\theta_{1}}\left(\theta_{1}>0\right)$ and exponential threshold $Y_{2}$ for cumulative wastage due to frequent breaks of existing workers with mean $\frac{1}{\theta_{2}}\left(\theta_{2}>0\right)$ and $\mathrm{Y}_{3}$ backup sources with mean $\frac{1}{\theta_{3}}\left(\theta_{3}>0\right)$. Let $\mathrm{q}(\mathrm{q} \neq 0)$ be the probability that every policy decision has exit of personnel. Let $\chi(I)$ be the indicator function of the event I. Let $\mathrm{T}$ be the random variable denoting the time to recruitment with mean $\mathrm{E}(\mathrm{T})$ and variance $\mathrm{V}(\mathrm{T})$.

Deviating from the conventional method of using Laplace transform, a different probabilistic analysis is employed here.

By the recruitment policy, recruitment is done whenever the cumulative wastage exceeds the threshold $\mathrm{Y}_{1}+\mathrm{Y}_{2}+\mathrm{Y}_{3}$. When the first decision is taken, recruitment would not have been done for $B_{1}$ units of time. If the wastage $X_{1}\left(=S_{1}\right)$ in the first exit epoch is greater than $Y_{1}+Y_{2}+Y_{3}$ then recruitment is done and in this case $T=B_{1}=D_{1}$.

However, if $S_{1} \leq Y_{1}+Y_{2}+Y_{3}$, the non-recruitment period will continue till the arrival of next exit epoch. If the cumulative sum $\mathrm{S}_{2}$ of wastage in the first two exit epochs exceeds $Y_{1}+Y_{2}+Y_{3}$, then recruitment is done and $T=B_{1}+B_{2}=D_{2}$.

If $S_{2} \leq Y_{1}+Y_{2}+Y_{3}$, then the non-recruitment period will continue till the arrival of next exit epoch. Depending on $S_{3}>Y_{1}+Y_{2}+Y_{3}$ or $S_{3} \leq Y_{1}+Y_{2}+Y_{3}$, recruitment is done or the nonrecruitment period continues and so on.

$$
\text { ie. } \mathrm{T}=\left\{\begin{array}{cc}
D_{1}, & \text { if } S_{1}>\mathrm{Y} 1+\mathrm{Y} 2+\mathrm{Y} 3 \\
D_{2}, & \text { if } S_{1} \leq \mathrm{Y} 1+\mathrm{Y} 2+\mathrm{Y} 3<S_{2} \\
\cdot \\
D_{i+1}, & \text { if } S_{i} \leq \mathrm{Y} 1+\mathrm{Y} 2+\mathrm{Y} 3<S_{i+1} \\
. & .
\end{array}\right.
$$

In terms of indicator function of an event, we can write $\mathrm{T}$ as $T=\sum_{i=0}^{\infty} D_{i+1} \chi\left(S_{i} \leq Y_{1}+Y_{2}+Y_{3}<S_{i+1}\right)$

(1) Taking expectation on both sides of (1) and using the result (for any event

L)

$\mathrm{E}(X \chi(L))=\mathrm{E}(\mathrm{X}) \mathrm{P}(\mathrm{L})$, we get

$E(T)=\sum_{i=0}^{\infty} E\left(D_{i+1}\right) P\left(s_{i} \leq Y_{1}+Y_{2}+Y_{3} \leq s_{i+1}\right)$

(2)

From (1) and from the definition of $\mathrm{D}_{\mathrm{i}+1}$, we get

$E(T)=\sum_{i=0}^{\infty} E\left(\sum_{k=1}^{i+1} B_{k}\right) P\left(s_{i} \leq Y_{1}+Y_{2}+Y_{3} \leq s_{i+1}\right)$

(3)

Since the inter-exit times $\mathrm{B}_{\mathrm{i}}$ 's are identically distributed, we write

$\mathrm{E}\left(\mathrm{B}_{\mathrm{i}}\right)=\mathrm{E}(\mathrm{B}), \mathrm{i}=1,2, \ldots$. Therefore from (3) we get

$E(T)=\sum_{i=0}^{\infty}(i+1) E(B) P\left(s_{i} \leq Y_{1}+Y_{2}+Y_{3} \leq s_{i+1}\right)$ 
By using law of total probability we get

$$
\begin{gathered}
P\left(S_{i} \leq Y_{1}+Y_{2}+Y_{3}<S_{i+1}\right)=P\left(0 \leq\left(Y_{1}+Y_{2}+Y_{3}\right)-S_{i}<X_{i+1}\right) \\
=\int_{0}^{\infty} \int_{0}^{t} P\left(0 \leq\left(Y_{1}+Y_{2}+Y_{3}\right)-S_{i}<X_{i+1} / S_{i}=x, Y_{1}+Y_{2}+Y_{3}\right. \\
=t)\left(e_{S_{i}, Y_{1}+Y_{2}+Y_{3}}(x, t)\right) d x d t \\
=\int_{0}^{\infty} \int_{0}^{t} P\left(X_{i+1}>t-x\right)\left(e_{S_{i}, Y_{1}+Y_{2}+Y_{3}}(x, t)\right) d x d t \\
=\int_{0}^{\infty} \int_{0}^{t} \tilde{M}(t-x) m_{i}(x) h(t) d x d t
\end{gathered}
$$

(5)

By hypothesis, $\tilde{M}(t-x)=e^{-\alpha(t-x)}$ and $m_{i}(x)=\left\{\frac{\alpha^{i} x^{i-1} e^{-\alpha x}}{\Gamma_{i}}\right\}$.

Therefore

$$
\begin{aligned}
P\left(S_{i} \leq Y_{1}+Y_{2}+Y_{3}<S_{i+1}\right) & =\int_{0}^{\infty}\left[\int_{0}^{t} e^{-\alpha(t-x)}\left\{\frac{\alpha^{i} x^{i-1} e^{-\alpha x}}{\Gamma i}\right\} h(t) d x d t\right] \\
& =\int_{0}^{\infty} \frac{e^{-\alpha t} \alpha^{i}}{\alpha^{i}(i-1) !}\left[\int_{0}^{t} x^{i-1} d x\right] h(t) d t \\
& =\int_{0}^{\infty} \frac{e^{-\alpha t} \alpha^{i} t^{i}}{i !} h(t) d t
\end{aligned}
$$

(6)

Since, for $\mathrm{i}=1,2,3 Y_{i} \sim \exp \left(\theta_{i}\right)$ and $Y_{i}^{\prime} s$ are independent, the probability density function $\mathrm{h}(\mathrm{t})$ of

$$
\mathrm{Y}_{1}+\mathrm{Y}_{2}+\mathrm{Y}_{3} \text { is found to be } \frac{\theta_{1} \theta_{2}}{\theta_{1}-\theta_{2}}\left[e^{-\theta_{2} t}-e^{-\theta_{1} t}+e^{-\left(\theta_{1}+\theta_{3}\right) t}-e^{-\left(\theta_{2}+\theta_{3}\right) t}\right]
$$

Therefore

$$
\begin{aligned}
& P\left(S_{i} \leq Y_{1}+Y_{2}+Y_{3}<S_{i+1}\right)=\int_{0}^{\infty} \frac{e^{-\alpha t} \alpha^{i} t^{i}}{i !} \frac{\theta_{1} \theta_{2}}{\theta_{1}-\theta_{2}}\left[e^{-\theta_{2} t}-e^{-\theta_{1} t}+e^{-\left(\theta_{1}+\theta_{3}\right) t}-\right. \\
& \left.e^{-\left(\theta_{2}+\theta_{3}\right) t}\right] d t \quad(7) \\
& P\left(S_{i} \leq Y_{1}+Y_{2}+Y_{3}<S_{i+1}\right)=\frac{\theta_{1} \theta_{2} \alpha^{i}}{\left(\theta_{1}-\theta_{2}\right) i !}\left[\frac{1}{\left(\alpha+\theta_{2}\right)^{i+1}}-\frac{1}{\left(\alpha+\theta_{1}\right)^{i+1}}+\frac{1}{\left(\alpha+\theta_{1}+\theta_{3}\right)^{i+1}}-\frac{1}{\left(\alpha+\theta_{2}+\theta_{3}\right)^{i+1}}\right]
\end{aligned}
$$

from (4) and (8) and on simplification it can be shown that

$E(T)=E(B) \sum_{i=0}^{\infty}(i+1) \frac{\theta_{1} \theta_{2} \alpha^{i}}{\left(\theta_{1}-\theta_{2}\right) i !}\left[\frac{1}{\left(\alpha+\theta_{2}\right)^{i+1}}-\frac{1}{\left(\alpha+\theta_{1}\right)^{i+1}}+\frac{1}{\left(\alpha+\theta_{1}+\theta_{3}\right)^{i+1}}-\frac{1}{\left(\alpha+\theta_{2}+\theta_{3}\right)^{i+1}}\right]$

(9)

We now find $E(B)$ for the present model.

The distribution function $G($.$) of the inter-exit times B_{i}, i=1,2, \ldots$ satisfy the relation 
$\mathrm{G}(\mathrm{x})=\mathrm{q} \sum_{n=1}^{\infty}(1-\mathrm{q})^{n-1} F_{n}(\mathrm{x})$

$$
\begin{aligned}
& E(B)=q \sum_{n=1}^{\infty}(1-q)^{n-1} E\left(A_{1}+A_{2}+\cdots+A_{n}\right) \\
& =q \sum_{n=1}^{\infty}(1-q)^{n-1}\left[\frac{a_{1} \lambda_{2}+a_{2} \lambda_{1}}{\lambda_{1} \lambda_{2}}+\frac{a_{1} \lambda_{2}+a_{2} \lambda_{1}}{\lambda_{1} \lambda_{2} c}+\frac{a_{1} \lambda_{2}+a_{2} \lambda_{1}}{\lambda_{1} \lambda_{2} c^{2}}+\cdots+\frac{a_{1} \lambda_{2}+a_{2} \lambda_{1}}{\lambda_{1} \lambda_{2} c^{n-1}}\right] \\
& =\frac{c q\left[a_{1} \lambda_{2}+a_{2} \lambda_{1}\right]}{\lambda_{1} \lambda_{2}(c-1)} \sum_{n=1}^{\infty}(1-q)^{n-1}-\frac{c q\left[a_{1} \lambda_{2}+a_{2} \lambda_{1}\right]}{\lambda_{1} \lambda_{2}(c-1) c} \sum_{n=1}^{\infty}\left[\frac{(1-q)}{c}\right]^{n-1} \\
& =\frac{c q\left[a_{1} \lambda_{2}+a_{2} \lambda_{1}\right]}{\lambda_{1} \lambda_{2}(c-1)}\left(\frac{1}{q}\right)-\frac{q\left[a_{1} \lambda_{2}+a_{2} \lambda_{1}\right]}{\lambda_{1} \lambda_{2}(c-1)}\left(\frac{c}{c-1+q}\right) \\
& =\frac{c\left[a_{1} \lambda_{2}+a_{2} \lambda_{1}\right]}{\lambda_{1} \lambda_{2}(c-1)}\left[\frac{c-1+q-q}{(c-1+q)}\right] \\
& E(B)=\frac{c\left[a_{1} \lambda_{2}+a_{2} \lambda_{1}\right]}{\lambda_{1} \lambda_{2}(c-1)}\left[\frac{(c-1)}{(c-1+q)}\right] \\
& \text { ie. } E(B)=\frac{c\left[a_{1} \lambda_{2}+a_{2} \lambda_{1}\right]}{\lambda_{1} \lambda_{2}(c-1+q)}
\end{aligned}
$$

Using (11) in (9), we get

$$
\begin{aligned}
E(T) & =\frac{c\left[a_{1} \lambda_{2}+a_{2} \lambda_{1}\right]}{\lambda_{1} \lambda_{2}(c-1+q)}\left[\frac{\alpha\left(\theta_{1}-\theta_{2}\right)+\theta_{1} \theta_{2}}{\theta_{1} \theta_{2}}-\frac{\theta_{1} \theta_{2} \alpha}{\left(\theta_{1}+\theta_{3}\right)\left(\theta_{2}+\theta_{3}\right)}\right] \\
E(T) & =N_{1} E(B)
\end{aligned}
$$

where $\mathrm{N}_{1}=\frac{\alpha\left(\theta_{1}-\theta_{2}\right)+\theta_{1} \theta_{2}}{\theta_{1} \theta_{2}}-\frac{\theta_{1} \theta_{2} \alpha}{\left(\theta_{1}+\theta_{3}\right)\left(\theta_{2}+\theta_{3}\right)}, \mathrm{E}(\mathrm{B})=\frac{c\left[a_{1} \lambda_{2}+a_{2} \lambda_{1}\right]}{\lambda_{1} \lambda_{2}(c-1+q)}$

We now evaluate $E\left(T^{2}\right)$ for the present model.

Squaring both sides on (2), we get

$$
\begin{gathered}
E\left(T^{2}\right)=\sum_{i=0}^{\infty} E\left(D_{i+1}^{2}\right) P\left(S_{i} \leq Y_{1}+Y_{2}+Y_{3}\right. \\
\left.<S_{i+1}\right) .
\end{gathered}
$$

Now

$$
\begin{aligned}
& E\left(D_{i+1}^{2}\right)=V\left(D_{i+1}\right)+\left[E\left(D_{i+1}\right)\right]^{2} \\
& \text { ie. } E\left(D_{i+1}^{2}\right)=(i+1) V(B)+[(i+1) E(B)]^{2} .
\end{aligned}
$$

Using (15) in (14), we get

$E\left(T^{2}\right)=\sum_{i=0}^{\infty}(i+1) V(B)\left[P\left(S_{i} \leq Y_{1}+Y_{2}+Y_{3}<S_{i+1}\right)\right]+\sum_{i=0}^{\infty}(i+1)^{2} E^{2}(B)\left[P\left(S_{i} \leq Y_{1}+Y_{2}+\right.\right.$ $\left.\left.Y_{3}<S_{i+1}\right)\right]$

We now evaluate $\mathrm{E}\left(\mathrm{B}^{2}\right)$ for the present model 
From (10) we get

$E\left(B^{2}\right)=\int_{0}^{\infty} x^{2} d G(x)=\int_{0}^{\infty} x^{2}\left[q \sum_{n=1}^{\infty}(1-q)^{n-1} f_{n}(x)\right] d x$

ie. $E\left(B^{2}\right)=q \sum_{n=1}^{\infty}(1-q)^{n-1} \int_{0}^{\infty} x^{2} f_{n}(x) d x$.

We now evaluate $\int_{0}^{\infty} x^{2} f_{n}(x) d x$ in (17).

$$
\begin{aligned}
& \int_{0}^{\infty} x^{2} f_{n}(x) d x=V\left(C_{n}\right)+\left[E\left(C_{n}\right)\right]^{2} \\
& \int_{0}^{\infty} x^{2} f_{n}(x) d x=\frac{c^{2}\left[2\left(a_{1} \lambda_{2}^{2}+a_{2} \lambda_{1}^{2}\right)-\left(a_{1} \lambda_{2}+a_{2} \lambda_{1}\right)^{2}\right]}{\left(c^{2}-1\right)\left(\lambda_{1} \lambda_{2}\right)^{2}}\left[\frac{c^{2 n}-1}{c^{2 n}}\right]+\left\{\frac{c\left[a_{1} \lambda_{2}+a_{2} \lambda_{1}\right]}{(c-1) \lambda_{1} \lambda_{2}}\left[\frac{c^{n}-1}{c^{n}}\right]\right\}^{2}
\end{aligned}
$$

Therefore from (18) we get

$$
\begin{gathered}
E\left(B^{2}\right)=\frac{c^{2} q\left[2\left(a_{1} \lambda_{2}^{2}+a_{2} \lambda_{1}^{2}\right)-\left(a_{1} \lambda_{2}+a_{2} \lambda_{1}\right)^{2}\right]}{\left(c^{2}-1\right)\left(\lambda_{1} \lambda_{2}\right)^{2}} \sum_{n=1}^{\infty}(1-q)^{n-1}\left[\frac{c^{2 n}-1}{c^{2 n}}\right] \\
+\frac{c^{2} q\left[a_{1} \lambda_{2}+a_{2} \lambda_{1}\right]^{2}}{(c-1)^{2}\left(\lambda_{1} \lambda_{2}\right)^{2}} \sum_{n=1}^{\infty}(1-q)^{n-1}\left[\frac{c^{n}-1}{c^{n}}\right]^{2} \\
=\frac{c^{2} q\left[2\left(a_{1} \lambda_{2}^{2}+a_{2} \lambda_{1}^{2}\right)-\left(a_{1} \lambda_{2}+a_{2} \lambda_{1}\right)^{2}\right]}{\left(c^{2}-1\right)\left(\lambda_{1} \lambda_{2}\right)^{2}}\left(\frac{1}{q}\right) \\
-\frac{c^{2} q\left[2\left(a_{1} \lambda_{2}^{2}+a_{2} \lambda_{1}^{2}\right)-\left(a_{1} \lambda_{2}+a_{2} \lambda_{1}\right)^{2}\right]}{c^{2}\left(c^{2}-1\right)\left(\lambda_{1} \lambda_{2}\right)^{2}}\left[\frac{c^{2}}{c^{2}-1+q}\right] \\
+\frac{c^{2} q\left[a_{1} \lambda_{2}+a_{2} \lambda_{1}\right]^{2}}{(c-1)^{2}\left(\lambda_{1} \lambda_{2}\right)^{2}}\left(\frac{1}{q}\right)+\frac{c^{2} q\left[a_{1} \lambda_{2}+a_{2} \lambda_{1}\right]^{2}}{c^{2}(c-1)^{2}\left(\lambda_{1} \lambda_{2}\right)^{2}} \sum_{n=1}^{\infty}\left[\frac{(1-q)}{c^{2}}\right]^{n-1} \\
-\frac{2 c^{2} q\left[a_{1} \lambda_{2}+a_{2} \lambda_{1}\right]^{2}}{c(c-1)^{2}\left(\lambda_{1} \lambda_{2}\right)^{2}} \sum_{n=1}^{\infty}\left[\frac{(1-q)}{c}\right]^{n-1} \\
=\frac{c^{2}\left[2\left(a_{1} \lambda_{2}^{2}+a_{2} \lambda_{1}^{2}\right)-\left(a_{1} \lambda_{2}+a_{2} \lambda_{1}\right)^{2}\right]}{\left(c^{2}-1+q\right)\left(\lambda_{1} \lambda_{2}\right)^{2}} \\
\left.+\frac{c^{2}\left[a_{1} \lambda_{2}+a_{2} \lambda_{1}\right]^{2}}{(c-1)^{2}\left(\lambda_{1} \lambda_{2}\right)^{2}} \frac{(c-1)^{2}[c+1-q]}{\left[c^{2}-1+q\right][c-1+q]}\right]^{2}+\frac{c^{2}\left[a_{1} \lambda_{2}+a_{2} \lambda_{1}\right]^{2}[c+1-q]}{\left(\lambda_{1} \lambda_{2}\right)^{2}\left[c^{2}-1+q\right][c-1+q]} \\
=\frac{2 c^{2}\left(a_{1} \lambda_{2}^{2}+a_{2} \lambda_{1}^{2}\right)-c^{2}\left(a_{1} \lambda_{2}+a_{2} \lambda_{1}\right)^{2}}{\left(c^{2}-1+q\right)\left(\lambda_{1} \lambda_{2}\right)^{2}}
\end{gathered}
$$

ie. $E\left(B^{2}\right)=\frac{2 c^{2}\left(a_{1} \lambda_{2}^{2}+a_{2} \lambda_{1}^{2}\right)}{\left(c^{2}-1+q\right)\left(\lambda_{1} \lambda_{2}\right)^{2}}$

From (8), (11) and (19), we get

$E\left(T^{2}\right)=E\left(B^{2}\right) N_{1}-\left[N_{1}-\left(N_{2}+N_{3}\right)\right] E^{2}(B)$

(20)

Where, 


$$
\begin{aligned}
& \mathrm{N}_{1}=\left[\frac{\left(\alpha\left(\theta_{1}-\theta_{2}\right)+\theta_{1} \theta_{2}\right.}{\theta_{1} \theta_{2}}-\frac{\theta_{1} \theta_{2} \alpha}{\left(\theta_{1}+\theta_{3}\right)\left(\theta_{2}+\theta_{3}\right)}\right] \\
& N_{2}=\frac{2 \alpha^{2}\left(\theta_{1}^{2}+\theta_{2}^{2}+\theta_{1} \theta_{2}\right)+3 \alpha \theta_{1} \theta_{2}\left(\theta_{1}+\theta_{2}\right)+\theta_{1}^{2} \theta_{2}^{2}}{\theta_{1}^{2} \theta_{2}^{2}} \\
& N_{3}= \\
& \frac{\theta_{1} \theta_{2}\left[\left(\theta_{1}^{5}-\theta_{2}^{5}\right)+\left(\theta_{1}^{4}-\theta_{2}^{4}\right)\left[3 \alpha+5 \theta_{3}\right]+\left(\theta_{1}^{3}-\theta_{2}^{3}\right)\left[2 \alpha^{2}+12 \alpha \theta_{3}+10 \theta_{3}^{2}\right]+\left(\theta_{1}^{2}-\theta_{2}^{2}\right)\left[6 \alpha^{2} \theta_{3}+6 \alpha \theta_{3}^{2}+10 \theta_{3}\right]+\left(\theta_{1}-\theta_{2}\right)\left[6 \alpha^{2} \theta_{3}^{2}+12 \alpha \theta_{3}^{3}+5 \theta_{3}^{4}\right]\right]}{\left(\theta_{1}+\theta_{3}\right)^{3}\left(\theta_{2}+\theta_{3}\right)^{3}}
\end{aligned}
$$

From (12) and (20) we get

$V(T)=E\left(B^{2}\right) N_{1}-E^{2}(B)\left[N_{1}^{2}+N_{1}-\left(N_{2}+N_{3}\right)\right]$

Where $E\left(B^{2}\right), E^{2}(B), N_{1}, N_{2}$ and $N_{3}$ represents from (11), (19) and (20) respectively

\section{CONCLUSION}

The problem of time to recruitment is studied in a more general setting on policy decision and brealdown threshold. Consequentlyn the process of planning of recruitment is more enlighted. Data analysis can be used to test the suitability of distributions assumed in the present work.

\section{REFERENCES}

[1] Bartholomew, D.J., "Statistical Model for Social Processes", New York, John Wiley and Sons, 1973.

[2] Bartholomew, D.J., and Andrew Forbes, F., "Statistical Techniques for Manpower Planning”, New York, John Wiley and Sons, 1979.

[3] Devi, A., and Srinivasan, A., "Variance of Time to Recruitment for a Single Grade Manpower System with Different Epochs for Decisions and Exits." International Journal of Research in Mathematics \& Computation, Vol.2, No.1, 2014, pp.23-27.

[4] Devi, A., and Srinivasan, A., "Probabilistic Analysis on Time to Recruitment for a Single Grade Manpower System with Different Epochs for Decisions and Exits. " International Journal of Revolution in Science and Humanity, Vol.4, No.2, 2014, pp.59-64.

[5] Devi, A., and Srinivasan, A., "Expected Time to Recruitment for a Single Grade Manpower System with Different Epochs for Decisions and Exits Having Inter-Decision Times as an Order Statistics. ” Mathematical Science- International Research Journal, Vol.3, No.2, 2014, pp.887890.

[6] Devi, A., and Srinivasan, A., "A Stochastic Model for Estimation of Variance of Time to Recruitment for a Single Grade Manpower System with Different Epochs for Decisions and Exits." International Journal of Applied Mathematics and Statistical Sciences, Vol.4, No.3, 2015, pp.1-8.

[7] Devi, A., and Srinivasan, A., "Expected Time to Recruitment for a Single Grade Manpower System with Different Epochs for Decisions and Exits with Inter-Decision Times as Order Statistics." International Journal of Innovative Research in Science, Engineering and Technology, Vol.4, No.8, 2015, pp.7517-7520.

[8] Devi, A., and Srinivasan, A., "Stochastic Models for Time to Recruitment in a Single Grade Manpower System with Non-instantaeneous Exits and Independent Wastage of Man Power 
when the Breakdown Threshold as Two Components Using Univariate CUM Policy of Recruitment." International Journal of management, IT and Engineering, Vol.9, No.1, 2019, pp.128-133.

[9] Ishwarya, G., Shivaranjani, N., and Srinivasan, A., "Expected Time to Recruitment in a Single Graded Manpower System with Inter-Decision Times as a Geometric Process.” Bonfring International Journal of ManMachine Interface, Vol.2, No.1, 2012, pp.7-10.

[10] Lalitha.R, Devi.A and Srinivasan. A, "A Stochastic Model on the Time to Recruitment for a Single Grade Manpower System with Attrition Generated by a Geometric Process of InterDecision Times Using Univariate Policy of Recruitment", Journal of Engineering Computers \&Applied Sciences, Vol.3, No.7, 2014, pp.12-15.

[11] Mariappan, P., Devi, A., and Srinivasan, A., "A Stochastic Model for Time to Recruitment in a Single Grade Manpower System with Different Epochs for Decisions and Exits Having Geometric Inter-Decision Times. " Second International Conference on Business Analytics and Intelligence, Bangalore, India, 2014.

[12] Muthaiyan, A., "A Study on Stochastic Models in Manpower Planning”, Ph.D Thesis, Bharathidasan University, Tiruchirappalli, 2010.

[13] Sathyamoorthi, R., and Elangovan, R., "Shock Model Approach to Determine the Expected Time to Recruitment." Journal of Decision and Mathematika Sciences, Vol.3, 1998, pp.67-78.

[14] Sheldon Ross, "A First Course in Probability", Sixth Edition, Published by Pearson Ltd, Indian branch, Patparganj, Delhi, India, 2004.

[15] Srinivasan, A., and Jayalakshmi, G., "Probabilistic Analysis on Time to Recruitment for a Single Grade Manpower System when the Breakdown Threshold has two Components." International Journal of Scientific Engineering and Applied Science, Vol.1, No.4, 2015, pp.180-183.

[16] Sridharan, J., Saivarajan, A., and Srinivasan, A., "Expected Time to Recruitment in a Single Graded Manpower System with Two Thresholds", Journal of Advances in Mathematics, Vol.7, 2014, pp.1147-1157.

[17] Vijayasankar, N., Elangovan, R., and Sathiyamoorthi, R., "Determination of Expected Time to Recruitment when Backup Resources of Manpower Exits. ” Ultra Scientist, Vol.25, No.1, 2013, pp.61-68. 